\title{
PAPER Portfolio Selection Models with Technical Analysis-Based Fuzzy Birandom Variables
}

\author{
You LI ${ }^{\dagger \mathrm{a})}$, Bo WANG ${ }^{\dagger \mathrm{b})}$, Nonmembers, and Junzo WATADA ${ }^{\dagger \mathrm{c})}$, Member
}

\begin{abstract}
SUMMARY Recently, fuzzy set theory has been widely employed in building portfolio selection models where uncertainty plays a role. In these models, future security returns are generally taken for fuzzy variables and mathematical models are then built to maximize the investment profit according to a given risk level or to minimize a risk level based on a fixed profit level. Based on existing works, this paper proposes a portfolio selection model based on fuzzy birandom variables. Two original contributions are provided by the study: First, the concept of technical analysis is combined with fuzzy set theory to use the security returns as fuzzy birandom variables. Second, the fuzzy birandom Value-at-Risk (VaR) is used to build our model, which is called the fuzzy birandom VaR-based portfolio selection model (FBVaR-PSM). The VaR can directly reflect the largest loss of a selected case at a given confidence level and it is more sensitive than other models and more acceptable for general investors than conventional risk measurements. To solve the FBVaR-PSM, in some special cases when the security returns are taken for trapezoidal, triangular or Gaussian fuzzy birandom variables, several crisp equivalent models of the FBVaR-PSM are derived, which can be handled by any linear programming solver. In general, the fuzzy birandom simulation-based particle swarm optimization algorithm (FBS-PSO) is designed to find the approximate optimal solution. To illustrate the proposed model and the behavior of the FBS-PSO, two numerical examples are introduced based on investors' different risk attitudes. Finally, we analyze the experimental results and provide a discussion of some existing approaches.

key words: portfolio selection, technical analysis, fuzzy birandom variable, Value-at-Risk, fuzzy birandom simulation, particle swarm optimization
\end{abstract}

\section{Introduction}

Portfolio selection theory aims at allocating capital to specific securities, so the investment can maximize profit or minimize risk. In 1952, Markowitz [1] used a single period variance of security returns as a risk measurement to develop techniques for portfolio optimization. Since then, various studies have been proposed in this field [2], [17], [25], [26].

The existing studies have mainly improved Markowitz's method in two aspects: the technique used to describe future security returns and the technique used to measure investment risk. The security returns in conventional models are generally determined by stochastic analysis based on precise historical data. However, on one hand, such precise data are not always available, e.g. for an IPO

Manuscript received December 20, 2012.

Manuscript revised September 5, 2013.

${ }^{\dagger}$ The authors are with the Graduate School of Information, Production and Systems, Waseda University, Kitakyushu-shi, 8080135 Japan.

a) E-mail: liyouwaseda@gmail.com

b)E-mail: bwangips@gmail.com

c)E-mail: junzow@ osb.att.ne.jp

DOI: $10.1587 /$ transinf.E97.D.11 (initial public offerings) share. On the other hand, the various inputs such as company performance, the market forces of supply and demand, positive and negative news about a company, and political factors, are necessary to form the basis for future return forecasting and are often assessed with some uncertainty. This type of uncertainty is usually nonstatistical, e.g. linguistic knowledge. In addition, forecasted values need to be adjusted by experts based on their confidence and knowledge about the future. These human estimations can also involve imprecise information. The probability theory is used for analyzing a greater amount of data, while fuzzy logic is used for the representation and use of linguistic knowledge. Therefore, many recent works (e.g. [2], [3], [9], [14], [17]) consider fuzzy set theory to be an alternative tool to stochastic theory when handling a security return forecast.

Risk measurements are of great importance in portfolio selection models, and various techniques have been developed to evaluate risk from different aspects. For example, the variance [1], Value-at-Risk (VaR) [6] and ChanceConstraints [18] are applied in stochastic portfolio selection problems. Mean-variance [9], mean-semivariance [3], mean-entropy [2] and the fuzzy VaR [17] are designed to build fuzzy portfolio selection models. These risk measurements can be divided into two types: The first type is used to enhance the stability of a portfolio, and it includes most of the existing approaches, such as variance, mean-variance and mean-entropy. The second type is designed to measure the exact loss of a portfolio, e.g. the VaR and fuzzy VaR. In our previous study [17], the VaR metric has been proven to be more effective and acceptable than other methods, because it can provide a more sensitive analysis of risk for investors and thus help them make decisions more easily. In this study, we extend the fuzzy VaR to a fuzzy birandom case, which is used as the risk measurement to build our model. We will provide a more in-depth explanation of this point in the following sections.

Peng [19] introduced the concept of a birandom variable in a stochastic environment. A birandom variable is defined as a binary random variable taking random values. In fuzzy set theory, the fuzzy birandom variable can be considered as a special case of the fuzzy random variable [20], when it includes only two fuzzy values.

Using the above knowledge, the technical analysis is applied in this study to forecast future security returns as fuzzy birandom variables. In finance, technical analysis is a security analysis discipline used for forecasting the direc- 
tion of prices through the study of past market data, primarily price and volume [22]. Related research [21] indicates that investors should identify recurring technical patterns, find laws and use this information to choose stocks. This methodology can greatly improve the performance of a portfolio. Therefore, in this research, we observe the historical prices of each stock studied and consider the times in a certain trading period at which prices fall and rise as a technical pattern. We first calculate the probabilities of upward and downward price changes, and we then forecast the future security returns for these stocks as fuzzy birandom variables.

The remainder of this paper is organized as follows: Section 2 introduces some basic information on fuzzy set theory, focusing on the fuzzy birandom variable and fuzzy birandom VaR. Section 3 explains the motivations behind our research. In Sect. 4, the fuzzy birandom Valueat-Risk-based portfolio selection model (FBVaR-PSM) is proposed, and some properties of the FBVaR-PSM are introduced. Section 5 introduces a general solution method to the FBVaR-PSM, called the fuzzy birandom simulationbased particle swarm optimization algorithm (FBS-PSO). In Sect. 6, we apply the proposed algorithm to solve two numerical examples and analyze the optimal results of the FBVaR-PSM. Finally Sect. 7 summarizes our conclusions.

\section{Preliminaries}

The fuzzy set theory has been applied in various fields to treat the endogenous fuzzily uncertainty. In this section, we first introduce some basic knowledge on the fuzzy variable and the fuzzy random variable, including the possibility, the necessary and credibility formulations. Then, the concept of the fuzzy birandom variable and the fuzzy birandom $\mathrm{VaR}$ are provided.

The fuzzy variable and fuzzy random variable are fundamental mathematical tools for describing fuzzy uncertainty.

Definition 2.1 ([8]). Suppose $\xi$ is a fuzzy variable whose membership function is $\mu_{\xi}$ and $r$ is a real number. Then, the credibility function of event $\xi \leq r$ can be expressed as follows:

$$
\operatorname{Cr}\{\xi \leq r\}=\frac{1}{2}[\operatorname{Pos}\{\xi \leq r\}+\operatorname{Nec}\{\xi \leq r\}],
$$

where $\mathrm{Cr}$ denotes the credibility of event $\xi \leq r$, Pos and $\mathrm{Nec}$ are the possibility and necessity measurements in possibility theory [4], defined as follows:

$$
\begin{aligned}
& \operatorname{Pos}\{\xi \leq r\}=\sup _{t \leq r} \mu_{\xi}(t), \\
& \operatorname{Nec}\{\xi \leq r\}=1-\sup _{t>r} \mu_{\xi}(t) .
\end{aligned}
$$

The credibility measurement is a self-dual set function [8], i.e., $\operatorname{Cr}\{\xi \leq r\}=1-\operatorname{Cr}\{\xi>r\}$, which describes the credibility of the events of fuzzy variables. If $\xi$ is taken as the fuzzy return of a security and $\operatorname{Cr}\{\xi \geq 5\}=0.8$, then we can say that the credibility of the event that the future return is no less than 5 is 0.8 .

Definition 2.2 ([13]). Suppose $\mathcal{L}$ is a fuzzy variable that represents the loss of one investment, then, the Valueat-Risk of $\mathcal{L}$ with a confidence of $(1-\beta)$ can be written as follows:

$$
\operatorname{VaR}_{1-\beta}=\sup \{\lambda \mid \operatorname{Cr}(\mathcal{L} \geq \lambda) \geq \beta\},
$$

where $\beta \in(0,1)$.

Equation (4) tells us that the greatest loss of $\mathcal{L}$ at confidence level $(1-\beta)$ is $\lambda$.

Definition 2.3 ([20]). A fuzzy random vector is a map $\xi=\left(\xi_{1}, \xi_{2}, \cdots, \xi_{n}\right): \Omega \rightarrow \mathcal{F}_{v}^{n}$ such that for any closed subset $F \subset \mathcal{R}^{n}, \operatorname{Pos}\{\gamma \mid \xi(\omega, \gamma) \in F\}$ is a $\Sigma$-measurable function of $\omega \in \Omega$, i.e., for any $t \in[0,1]$, we have

$$
\{\omega \in \Omega \mid \operatorname{Pos}\{\gamma \mid \xi(\omega, \gamma) \in F\} \leq t\} \in \Sigma .
$$

$(\Omega, \Sigma, \operatorname{Pr})$ is a probability space and $\mathcal{F}_{v}^{n}$ is a collection of fuzzy vectors defined on a possibility space ( $\Gamma, \mathcal{A}, \mathrm{Pos}), \xi$ is called a fuzzy random variable when $n=1$.

Definition 2.4 ([20]). Assume that $\xi$ is a fuzzy random variable defined on a probability space $(\Omega, \Sigma, \operatorname{Pr})$, the expected value of $\xi$ is defined as follows

$$
\begin{aligned}
& \mathrm{E}[\xi]= \\
& \int_{\Omega}^{\infty}\left[\int_{0}^{\infty} \mathrm{Cr}\{\xi(\omega) \geq r\} \mathrm{d} r-\int_{-\infty}^{0} \mathrm{Cr}\{\xi(\omega) \leq r\} \mathrm{d} r\right] \operatorname{Pr}(\mathrm{d} \omega),
\end{aligned}
$$

where $\operatorname{Pr}$ is the probability measurement and $\mathrm{E}$ is the expected value operator.

Definition 2.5 ([23]). Let $\xi$ be a fuzzy random variable, and $B$ a Borel subset of $\mathcal{R}$. The mean chance of an event $\xi \in B$ is defined as

$$
\operatorname{Ch}\{\xi \in B\}=\int_{0}^{1} \operatorname{Pr}\{\omega \in \Omega \mid \operatorname{Cr}\{\xi(\omega) \in B\} \geq r\} \mathrm{d} r,
$$

where $\mathrm{Ch}$ is the mean chance measurement.

Definition 2.6 ([12]). Suppose $\mathcal{L}$ is a fuzzy random variable that represents the loss of one investment, then, the VaR of $\mathcal{L}$ with a confidence of $(1-\beta)$ (in the sense of mean chance) is given by:

$$
\operatorname{VaR}_{1-\beta}=\sup \{\lambda \in \mathcal{R} \mid \operatorname{Ch}(\mathcal{L} \geq \lambda) \geq \beta\},
$$

where $\beta \in(0,1)$, and $\mathrm{Ch}$ is the mean chance measurement in Eq. (7).

Equation (8) tells us that the greatest loss of $\mathcal{L}$ under mean chance $(1-\beta)$ is $\lambda$.

Based on Definition 2.3, we can obtain the concept of fuzzy birandom variable easily, detailed as follows:

Definition 2.7 A fuzzy birandom variable is a map $\xi=$ $\left(\xi_{1}, \xi_{2}\right): \Omega \rightarrow \mathcal{F}_{v}^{n}$ such that for any closed Borel subset $F \subset \mathcal{R}^{n}, \operatorname{Pos}\{\gamma \mid \xi(\omega, \gamma) \in F\}$ is a $\Sigma$-measurable with respect to $\omega$. As $n=1, \xi$ is called a fuzzy birandom variable.

Then, the expected value, mean chance and Value-atRisk of the birandom variable can be obtained easily based 
Table 1 The comparisons of the fuzzy VaR and existing risk measurements.

\begin{tabular}{ccc}
\hline Methods Name & Risk Measurement Function & Purpose \\
\hline Mean-risk [11] & $\mathrm{Cr}\left\{b-\left(x_{1} \xi_{1}+x_{2} \xi_{2}+\cdots+x_{n} \xi_{n}\right) \geq r\right\} \leq \alpha(r)$ & $\begin{array}{c}\text { Describe the credibility values of } \\
\text { the bad events, which should lower } \\
\text { than the given tolerance levels }\end{array}$ \\
\hline Mean-variance [9] & $\mathrm{V}\left[x_{1} \xi_{1}+x_{2} \xi_{2}+\cdots+x_{n} \xi_{n}\right] \leq r$ & $\begin{array}{c}\text { Reflects an average deviation level } \\
\text { from the expected value, which } \\
\text { is limited by the given vale }\end{array}$ \\
\hline Mean-semivariance [3] & $\mathrm{SV}\left[x_{1} \xi_{1}+x_{2} \xi_{2}+\cdots+x_{n} \xi_{n}\right] \leq r$ & $\begin{array}{c}\text { Measures only the } \\
\text { lower deviation from } \\
\text { the expected return }\end{array}$ \\
\hline Entropy optimization [2] & $\mathrm{H}\left[x_{1} \xi_{1}+x_{2} \xi_{2}+\cdots+x_{n} \xi_{n}\right] \leq r$ & $\begin{array}{c}\text { Minimize the entropy can reduce } \\
\text { the uncertainty of a portfolio and } \\
\text { make the prediction easily }\end{array}$ \\
\hline Mean-variance-skewness [14] & $\mathrm{V}\left[x_{1} \xi_{1}+x_{2} \xi_{2}+\cdots+x_{n} \xi_{n}\right] \leq \alpha$ & $\begin{array}{c}\text { Reflects an average deviation } \\
\text { level from the expected value } \\
\text { with asymmetry fuzzy returns }\end{array}$ \\
\hline Fuzzy Value-at-Risk [17] & $\mathrm{S}\left[x_{1} \xi_{1}+x_{2} \xi_{2}+\cdots+x_{n} \xi_{n}\right] \geq \beta$ & $\begin{array}{c}\text { Directly reflect the loss } \\
\text { of one selection case under } \\
\text { given confidence level }\end{array}$ \\
\hline & $\operatorname{VaR}{ }_{1-\beta}=\sup \{\lambda \mid \operatorname{Cr}(\mathcal{L} \geq \lambda) \geq \beta\}$ &
\end{tabular}

on Definition 2.4, 2.5 and 2.6. We use the following example to help readers understand this point.

Example 2.1. Let $\Omega=\left(\omega_{1}, \omega_{2}\right), \mathrm{P}_{r}\left(\omega_{1}\right)=\mathrm{P}^{1}, \mathrm{P}_{r}\left(\omega_{2}\right)=$ $\mathrm{P}^{2}$ and $\mathrm{P}^{1}+\mathrm{P}^{2}=1$. Assume that $\xi^{1,2}$ is a fuzzy birandom variable defined on a possibility space $(\Gamma, \mathcal{A}, \mathrm{Pos})$, then,

$$
\xi(\omega)= \begin{cases}\xi^{1}, & \text { if } \omega=\omega_{1} \\ \xi^{2}, & \text { if } \omega=\omega_{2}\end{cases}
$$

where, $\xi^{1}$ and $\xi^{2}$ are fuzzy variables.

Now, we calculate the expected value, mean chance and Value-at-Risk of $\xi^{1,2}$. Results are listed as follows:

$$
\begin{aligned}
\mathrm{E}\left[\xi^{1,2}\right]= & \\
\mathrm{P}^{1} * & {\left[\int_{0}^{\infty} \operatorname{Cr}\left\{\xi^{1} \geq r\right\} \mathrm{d} r-\int_{-\infty}^{0} \operatorname{Cr}\left\{\xi^{1} \leq r\right\} \mathrm{d} r\right] } \\
+\mathrm{P}^{2} * & {\left[\int_{0}^{\infty} \operatorname{Cr}\left\{\xi^{2} \geq r\right\} \mathrm{d} r-\int_{-\infty}^{0} \operatorname{Cr}\left\{\xi^{2} \leq r\right\} \mathrm{d} r\right], } \\
\operatorname{Ch}\left\{\xi^{1,2} \in B\right\}= & \mathrm{P}^{1} *\left\{\omega_{1} \in \Omega \mid \operatorname{Cr}\left\{\xi^{1}\left(\omega_{1}\right) \in B\right\} \geq r\right\} \\
+ & \mathrm{P}^{2} *\left\{\omega_{2} \in \Omega \mid \operatorname{Cr}\left\{\xi^{2}\left(\omega_{2}\right) \in B\right\} \geq r\right\}, \\
\operatorname{VaR}_{1-\beta}= & \mathrm{P}^{1} * \sup \left\{\lambda_{1} \mid \operatorname{Ch}\left(\xi^{1} \geq \lambda_{1}\right) \geq \beta\right\} \\
+ & \mathrm{P}^{2} * \sup \left\{\lambda_{2} \mid \operatorname{Ch}\left(\xi^{2} \geq \lambda_{2}\right) \geq \beta\right\} .
\end{aligned}
$$

\section{Motivations}

\subsection{The Advantages of Fuzzy VaR}

The VaR of an investment is the likelihood of the greatest loss at a given confidence level [5]. It is defined with respect to a specific portfolio of financial assets with a specified probability over a specified time horizon. Recently, the VaR has been introduced to the fuzzy environment to build a fuzzy portfolio selection model [17].

Some other methods have been used to build fuzzy portfolio selection models such as mean-variance, meansemivariance and mean-entropy. Table 1 lists these risk measurements along with the fuzzy VaR.

From Table 1, we can find the difference between the fuzzy VaR and other approaches. Most of the existing models find an optimal solution by minimizing the variance or entropy, thus maximizing the stability of a portfolio. These models do not focus on the risk of exact future loss, which is sensitive consideration for general investors. However, the fuzzy VaR can identify how much return the investors can expect and how much future loss they may suffer in the worst case scenario.

One example explains the difference clearly. In the mean entropy optimization model, if the risk level $r$ (entropy value) is modified from 1.6 to 1.7 , then investors can obtain a selection case with a higher expected return. In this case, however, it is quite difficult for them to determine which portfolio to select. Because investors cannot identify how much higher the future risk becomes as the entropy increases by 0.1 , they cannot balance the higher expected return with the higher risk; however with the fuzzy VaR, investors can determine the confidence level and the expected return with more precision, and any change in these values will be directly reflected by the greatest possible loss obtained from the fuzzy VaR. Therefore, from this perspective, the portfolio selection model based on the fuzzy VaR can provide more sensitive information for measuring the future risk for investors and help them make decisions more easily.

Recently, the theory of Value-at-Risk has been applied to many engineering problems to build the fuzzy optimization models, to which the readers may refer [10], [12], [13], [15], [16].

\subsection{Describing the Future Security Returns as Fuzzy Bi- random Variables}

In this study, we first observe the historical prices of each stock selected, considering the times at which prices rise and fall in a certain trading period as a technical pattern. We then calculate the probabilities of the rises and falls, and forecast the security future returns as fuzzy birandom variables. This processes can be summarized by the following steps: 
[Step 1]. Observe the historical prices of each candidate stock and record the rising and falling times separately for each stock.

[Step 2]. Calculate the probabilities of the fall $\left(\mathrm{P}^{1}\right)$ and rise $\left(\mathrm{P}^{2}\right)$ for each stock.

[Step 3]. Forecast the future prices as two fuzzy variables based on the historical data: One is a loss variable $\left(\xi^{1}\right)$ which is the price decreasing interval. Another is a profit variable $\left(\xi^{2}\right)$ which indicates the stock price will increase within this interval.

[Step 4]. Use the security future return as a fuzzy birandom variable, which includes two fuzzy variables $\xi^{1}$ and $\xi^{2}$ with probabilities of $\mathrm{P}^{1}$ and $\mathrm{P}^{2}$.

[Step 5]. Adjust the forecast data using expert opinions from the related fields, if possible. An expert can revise the fall/rise probabilities or the fuzzy loss/profit variables according to his attitudes to the social policies, industry performance, company news and other factors which will affect the future return of the security.

The advantage of this approach is two-folded. First, compared with existing fuzzy portfolio selection models, this method uses technical analysis to divide conventional fuzzy variable into two vectors. This behavior can enhance the accuracy of forecasting, as each vector represents only the increment or decrement of the security return. Second, the proposed method can simplify the complexity of the fuzzy random optimizations, for both forecasting and solving.

\section{The Fuzzy Birandom Value-at-Risk-Based Portfolio Selection Model}

\subsection{Conventional Models with Fuzzy VaR}

The VaR has been applied to build the fuzzy portfolio selection model, in which the future security returns are described as fuzzy variables [17]. Supposing $p_{i}^{\prime}$ is the estimated closing prices of the securities $i(i=1,2, \cdots, n)$ in the future, $p_{i}$ are the closing prices at present, and $d_{i}$ are the estimated dividends of securities $i$ for an indefinite period of time, then the return of security $i$ is defined by the fuzzy variable $\xi_{i}$ as $\xi_{i}=\left(p_{i}^{\prime}+d_{i}-p_{i}\right) / p_{i}$. The mathematical model is introduced as follows:

$$
\left\{\begin{array}{l}
\max \mathrm{E}\left[x_{1} \xi_{1}+x_{2} \xi_{2}+\cdots+x_{n} \xi_{n}\right] \\
\text { Subject to } \\
\quad \operatorname{VaR}_{1-\beta}=\sup \{\lambda \mid \operatorname{Cr}(\mathcal{L} \geq \lambda) \geq \beta\} \leq S \\
\quad x_{1}+x_{2}+\cdots+x_{n}=1, \\
\quad x_{i} \geq 0, i=1,2, \cdots, n
\end{array}\right.
$$

or,

$$
\left\{\begin{array}{l}
\min \operatorname{VaR}_{1-\beta}=\sup \{\lambda \mid \operatorname{Cr}(\mathcal{L} \geq \lambda) \geq \beta\} \\
\text { Subject to } \\
\mathrm{E}\left[x_{1} \xi_{1}+x_{2} \xi_{2}+\cdots+x_{n} \xi_{n}\right] \geq R \\
x_{1}+x_{2}+\cdots+x_{n}=1 \\
x_{i} \geq 0, i=1,2, \cdots, n
\end{array}\right.
$$

where $S$ is the largest loss that an investor can accept, $R$

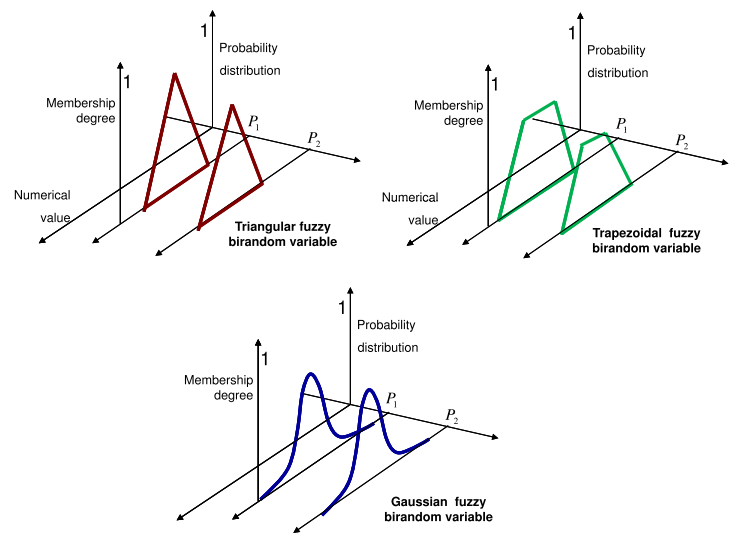

Fig. 1 Frequently-used fuzzy birandom variables.

is the given level of return, $\mathrm{VaR}_{1-\beta}$ indicates the greatest loss at $(1-\beta)$ confidence level, $\mathrm{E}\left[x_{1} \xi_{1}+x_{2} \xi_{2}+\cdots+x_{n} \xi_{n}\right]$ is the expected return and $\mathcal{L}$ is the loss function as $\mathcal{L}=$ $-\left(x_{1} \xi_{1}+x_{2} \xi_{2}+\cdots+x_{n} \xi_{n}\right)$. Model (13) is developed for risk-averters and model (14) is built for risk-lovers.

\subsection{The FBVaR-PSM}

The security returns in this research are used as fuzzy birandom variables, and the proposed model FBVaR-PSM is obtained as below:

$$
\left\{\begin{array}{l}
\max \mathrm{E}\left[x_{1} \xi_{1}^{1,2}+x_{2} \xi_{2}^{1,2}+\cdots+x_{n} \xi_{n}^{1,2}\right] \\
\text { Subject to } \\
\quad \operatorname{VaR}_{1-\beta}=\sup \{\lambda \mid \operatorname{Ch}(\mathcal{L} \geq \lambda) \geq \beta\} \leq S \\
x_{1}+x_{2}+\cdots+x_{n}=1 \\
x_{i} \geq 0, i=1,2, \cdots, n
\end{array}\right.
$$

or,

$$
\left\{\begin{array}{l}
\min \operatorname{VaR}_{1-\beta}=\sup \{\lambda \mid \operatorname{Ch}(\mathcal{L} \geq \lambda) \geq \beta\} \\
\text { Subject to } \\
\quad \mathrm{E}\left[x_{1} \xi_{1}^{1,2}+x_{2} \xi_{2}^{1,2}+\cdots+x_{n} \xi_{n}^{1,2}\right] \geq R \\
x_{1}+x_{2}+\cdots+x_{n}=1 \\
x_{i} \geq 0, i=1,2, \cdots, n
\end{array}\right.
$$

Models (15) and (16) are built for risk-averters and risk-lovers respectively. The future return of each security $i$ includes two fuzzy variables $\xi_{i}^{1}$ and $\xi_{i}^{2}$, the probabilities of which are determined by the rise and fall times of a stock historically. The fuzzy birandom VaR is calculated using the mean chance measurement $(\mathrm{Ch})$ instead of the credibility measurement $(\mathrm{Cr})$.

\subsection{Some Properties of FBVaR-PSM}

In special situations, when the portfolio returns can be used to represent the same distributed fuzzy birandom variables, such as trapezoidal, triangular or Gaussian fuzzy birandom variables, as listed in Fig. 1, the following theorems enable us to solve the models easily.

Theorem 1. Supposing the security returns are independent Gaussian fuzzy birandom variables with membership function $\mu_{\xi_{i}^{1,2}}(x)=\operatorname{Exp}\left\{-\left[\left(x_{i}-a_{i}^{1,2}\right) / \sigma_{i}^{1,2}\right]^{2}\right\}$, denoted 
as $\xi_{i}^{1,2}=\mathrm{FN}\left(a_{i}^{1,2}, \sigma_{i}^{1,2}\right)$, and $a_{i}^{1} \leq a_{i}^{2}$. The probability of each birandom variable is $\mathrm{P}_{i}^{1,2}$, the investment proportions of each security $i$ is $x_{i}$. Then for any portfolio selection problem, the model (16) is transformed to (17) and (18):

Proof. Equations (19) and (20) calculate the possibility of $\xi_{i}^{1,2}$ :

Therefore, from Eqs. (1) and (6) we obtain the credibility and expected value of $\xi_{i}^{1,2}=\operatorname{FN}\left(a_{i}^{1,2}, \sigma_{i}^{1,2}\right)$ with probabilities of $\mathrm{P}_{i}^{1}$ and $\mathrm{P}_{i}^{2}$, as in Eqs. (21) and (22):

According to Eqs. (8) and (21), the $\mathrm{VaR}_{1-\beta}$ can be computed in Eq. (24):

In fuzzy set theory, Eq. (25) is given for $\mathrm{n}$ th independent normal distribution fuzzy variables: (27).

Therefore, we obtain the conclusions of Eqs. (26) and

According to different confidence levels, the FBVaRPSM has the forms (17) and (18), respectively.

The proof is completed.

Theorem 2. Supposing all the portfolios' returns can be taken for the independent trapezoidal fuzzy birandom variables as $\xi_{i}^{1,2}=\left(a_{i}^{1,2}, b_{i}^{1,2}, c_{i}^{1,2}, d_{i}^{1,2}\right)$, the probability of each birandom variable is $\mathrm{P}_{i}^{1,2}$, and denote the investment proportions of each security $i$ as $x_{i}$, then for any portfolio selection, the model (16) results in (28) and (29):

Proof. Considering the portfolio returns are independent, then we have Eq. (30).

Based on Eq. (10), the expected value of any fuzzy birandom variable can be calculated by Eq. (31).

Therefore, we are able to compute the expected value of $\sum_{i=1}^{n} x_{i} \xi_{i}^{1,2}$, as in Eq. (32).

Now we compute the $\mathrm{VaR}_{1-\beta}$. This calculation is similar as in the proof of theorem 1 , and the result is obtained as follows:

According to Eqs. (11) and (12), the VaR function of $\mathcal{L}=-\left(x_{1} \xi_{1}^{1,2}+x_{2} \xi_{2}^{1,2}+\cdots+x_{n} \xi_{n}^{1,2}\right)$ is described in Eq. (33):

Considering different confidence levels, the FBVaRPSM has the forms (28) and (29), respectively.

The proof is completed.

Remark 1: In Theorem 2, when $b_{i}^{1,2}=c_{i}^{1,2}$, these trapezoidal independent fuzzy variables change to a triangular one. Therefore, for any triangular independent fuzzy values $\xi_{i}=\left(a_{i}^{1,2}, b_{i}^{1,2}, c_{i}^{1,2}\right)$, the model (16) equals to (34) or (35)

The updated models (17), (18), (28), (29), (34) and (35) are linear programming problems that can be solved directly. Similarly, after some considerations, we can also obtain similar theorems on model (15).

\section{Solution}

This section provides the solution method FBS-PSO for the proposed models. In Eq. (12), we discussed the calculation of the VaR of a single fuzzy birandom variable. However, our research must address a series of differently distributed fuzzy birandom variables, such as $\xi=\left(\xi_{1}^{1,2}, \xi_{2}^{1,2}, \cdots, \xi_{n}^{1,2}\right)$, the potential fuzzy loss of which should be considered as $\mathcal{L}=-\left[x_{1} \xi_{1}^{1,2}+x_{2} \xi_{2}^{1,2}+\cdots+x_{n} \xi_{n}^{1,2}\right]$, and in which it is impossible to solve such a problem $\operatorname{VaR}_{1-\beta}\left(x_{i}\right)=\sup \{\lambda \mid \operatorname{Ch}(\mathcal{L} \geq$ $\lambda) \geq \beta$ \} by definition. Therefore, we employ a fuzzy birandom simulation to calculate the VaR and combine the calculation with particle swarm optimization algorithm to find the approximate optimal solution. The proposed method is called the fuzzy birandom simulation-based particle swarm optimization algorithm (FBS-PSO).

\subsection{The Fuzzy Birandom Simulation}

The fuzzy birandom simulation is an extended version of the fuzzy simulation developed by Liu [24]. This method plays a pivotal role in solving the difficulty discussed above for solving VaR when there are differently distributed fuzzy birandom varaibles. Employing this technique, the theoretical calculation of fuzzy birandom VaR can be briefly summarized as follows:

Suppose $\xi=\left(\xi_{1}^{1,2}, \xi_{2}^{1,2}, \cdots, \xi_{n}^{1,2}\right)$ is a series of fuzzy birandom variables with support $\prod_{i=1}^{n}\left[a_{i}^{L_{1}, L_{2}}, a_{i}^{U_{1}, U_{2}}\right]$, where $a_{i}^{L_{1}, L_{2}}$ and $a_{i}^{U_{1}, U_{2}}$ are the lower and upper supports of $\xi_{i}^{1,2}$. $a_{i}$ and $b_{i}$ are the probabilities of fuzzy variables $\xi_{i}^{1}$ and $\xi_{i}^{2}$.

First, we divide each fuzzy birandom variable into $l$ parts $\zeta_{i}^{r_{1}}, \zeta_{i}^{r_{2}}$ which are constructed in Eq. (36):

$$
\left\{\begin{array}{l}
\zeta_{i}^{r_{1}}=a_{i}^{L_{1}}+\frac{r_{1}}{l}\left(a_{i}^{U_{1}}-a_{i}^{L_{1}}\right) \\
\zeta_{i}^{r_{2}}=a_{i}^{L_{2}}+\frac{r_{2}}{l}\left(a_{i}^{U_{2}}-a_{i}^{L_{2}}\right),
\end{array}\right.
$$

where, $r_{1}, r_{2}$ and $l$ are integers, and $0 \leq r_{1} \leq l, 0 \leq r_{2} \leq l$.

Second, we approximate the membership function of $\mu_{\xi}$ by a sequence of discrete fuzzy vectors $\left(a * \zeta_{i}^{r_{1}}+b * \zeta_{i}^{r_{2}}\right)$.

Third, the loss variable $\mathcal{L}=-\left(x_{1} \xi_{1}^{1,2}+x_{2} \xi_{2}^{1,2}+\cdots+\right.$ $\left.x_{n} \xi_{n}^{1,2}\right)$ can also be simulated by the fuzzy vectors $\left(a * \zeta_{i}^{r_{1}}+\right.$ $\left.b * \zeta_{i}^{r_{2}}\right)$.

Forth, based on the approximate membership function of $\mathcal{L}$, the mean chance measurements $\operatorname{Ch}(\mathcal{L} \geq \lambda)$ and $\mathrm{Ch}(\mathcal{L}<\lambda)$ can be calculated according to Eq. (7).

Finally, we can obtain an approximate value of fuzzy birandom VaR using the Eq. (8).

Based on the above procedures, we can say that a larger value of $l$ results in a more accurate result, but a longer computation time. Therefore, it can be concluded that the value of $l$ should vary based on the optimization problems being considered.

\subsection{The Improved Particle Swarm Optimization Algo- rithm}

The fuzzy birandom simulation is combined with the particle swarm optimization algorithm (PSO) to solve the FBVaR-PSM. The PSO algorithm was initially proposed by Kennedy and Eberhart [7] in 1995. This algorithm uses collaboration among a population of simple search agents 


$$
\left\{\begin{array}{l}
\min \sum_{i=1}^{n} x_{i}\left[\left(\mathrm{P}_{i}^{1} * a_{i}^{1}+\mathrm{P}_{i}^{2} * a_{i}^{2}\right)+(\right. \\
\text { Subject to } \\
\sum_{i=1}^{n} x_{i}\left(\mathrm{P}_{i}^{1} * a_{i}^{1}+\mathrm{P}_{i}^{2} * a_{i}^{2}\right) \geq R \\
x_{1}+x_{2}+\cdots+x_{n}=1 \\
x_{i} \geq 0, i=1,2, \cdots, n
\end{array}\right.
$$

$\beta>0.5$,

$$
\left\{\begin{array}{l}
\min \sum_{i=1}^{n} x_{i}\left\{\left(\mathrm{P}_{i}^{1} * a_{i}^{1}+\mathrm{P}_{i}^{2} * a_{i}^{2}\right)+\left(\mathrm{P}_{i}^{1} * \sigma_{i}^{1}+\mathrm{P}_{i}^{2} * \sigma_{i}^{2}\right) \sqrt{\ln [1 /(2-2 \beta)]}\right\} \\
\text { Subject to } \\
\quad \sum_{i=1}^{n} x_{i}\left(\mathrm{P}_{i}^{1} * a_{i}^{1}+\mathrm{P}_{i}^{2} * a_{i}^{2}\right) \geq R \\
x_{1}+x_{2}+\cdots+x_{n}=1 \\
x_{i} \geq 0, i=1,2, \cdots, n .
\end{array}\right.
$$$$
\begin{aligned}
& \operatorname{Pos}\left(x_{i} \geq r\right)= \begin{cases}1 & \mathrm{r}<a_{i}^{1} \\
\mathrm{P}_{i}^{1} * \operatorname{Exp}\left\{-\left[\left(r-a_{i}^{1}\right) / \sigma_{i}^{1}\right]^{2}\right\}+\mathrm{P}_{i}^{2} & a_{i}^{1} \leq \mathrm{r} \leq a_{i}^{2} \\
\mathrm{P}_{i}^{1} * \operatorname{Exp}\left\{-\left[\left(r-a_{i}^{1}\right) / \sigma_{i}^{1}\right]^{2}\right\}+\mathrm{P}_{i}^{2} * \operatorname{Exp}\left\{-\left[\left(r-a_{i}^{2}\right) / \sigma_{i}^{2}\right]^{2}\right\} & \mathrm{r}>\mathrm{a}_{i}^{2}\end{cases} \\
& \operatorname{Pos}\left(x_{i}<r\right)= \begin{cases}1 & \mathrm{r}>a_{i}^{2} \\
P_{i}^{1}+\mathrm{P}_{i}^{2} * \operatorname{Exp}\left\{-\left[\left(r-a_{i}^{2}\right) / \sigma_{i}^{2}\right]^{2}\right\} & a_{i}^{1} \leq \mathrm{r} \leq a_{i}^{2} \\
\mathrm{P}_{i}^{1} * \operatorname{Exp}\left\{-\left[\left(r-a_{i}^{1}\right) / \sigma_{i}^{1}\right]^{2}\right\}+\mathrm{P}_{i}^{2} * \operatorname{Exp}\left\{-\left[\left(r-a_{i}^{2}\right) / \sigma_{i}^{2}\right]^{2}\right\} & \mathrm{r}<\mathrm{a}_{i}^{1}\end{cases} \\
& \operatorname{Cr}\left\{\xi_{i}^{1,2} \geq r\right\}= \begin{cases}1-\frac{1}{2}\left(\mathrm{P}_{i}^{1} * \operatorname{Exp}\left\{-\left[\left(r-a_{i}^{1}\right) / \sigma_{i}^{1}\right]^{2}\right\}+\mathrm{P}_{i}^{2} * \operatorname{Exp}\left\{-\left[\left(r-a_{i}^{2}\right) / \sigma_{i}^{2}\right]^{2}\right\}\right) & \mathrm{r}<\mathrm{a}_{i}^{1} \\
\frac{1}{2}\left(1+\mathrm{P}_{i}^{2}-\mathrm{P}_{i}^{1}+\mathrm{P}_{i}^{1} * \operatorname{Exp}\left\{-\left[\left(r-a_{i}^{1}\right) / \sigma_{i}^{1}\right]^{2}\right\}-\mathrm{P}_{i}^{2} * \operatorname{Exp}\left\{-\left[\left(r-a_{i}^{2}\right) / \sigma_{i}^{2}\right]^{2}\right\}\right) & a_{i}^{1} \leq \mathrm{r} \leq a_{i}^{2} . \\
\frac{1}{2}\left(\mathrm{P}_{i}^{1} * \operatorname{Exp}\left\{-\left[\left(r-a_{i}^{1}\right) / \sigma_{i}^{1}\right]^{2}\right\}+\mathrm{P}_{i}^{2} * \operatorname{Exp}\left\{-\left[\left(r-a_{i}^{2}\right) / \sigma_{i}^{2}\right]^{2}\right\}\right) & \mathrm{r}>\mathrm{a}_{i}^{2} .\end{cases}
\end{aligned}
$$

$\mathrm{E}\left[\xi_{i}^{1,2}\right]=\mathrm{P}_{i}^{1} *\left[\int_{0}^{\infty} \mathrm{Cr}\left\{\xi_{i}^{1} \geq r\right\} \mathrm{d} r-\int_{-\infty}^{0} \operatorname{Cr}\left\{\xi_{i}^{1} \leq r\right\} \mathrm{d} r\right]+\mathrm{P}^{2} *\left[\int_{0}^{\infty} \operatorname{Cr}\left\{\xi_{i}^{2} \geq r\right\} \mathrm{d} r-\int_{-\infty}^{0} \operatorname{Cr}\left\{\xi_{i}^{2} \leq r\right\} \mathrm{d} r\right]$.

$$
\mathrm{E}[\xi]=\mathrm{P}_{i}^{1} * a_{i}^{1}+\mathrm{P}_{i}^{2} * a_{i}^{2}
$$

$$
\operatorname{VaR}_{1-\beta}= \begin{cases}\left(\mathrm{P}_{i}^{1} * a_{i}^{1}+\mathrm{P}_{i}^{2} * a_{i}^{2}\right)+\left(\mathrm{P}_{i}^{1} * \sigma_{i}^{1}+\mathrm{P}_{i}^{2} * \sigma_{i}^{2}\right) \sqrt{\ln (1 / 2 \beta)} & 0<\beta \leq 0.5 \\ \left(\mathrm{P}_{i}^{1} * a_{i}^{1}+\mathrm{P}_{i}^{2} * a_{i}^{2}\right)+\left(\mathrm{P}_{i}^{1} * \sigma_{i}^{1}+\mathrm{P}_{i}^{2} * \sigma_{i}^{2}\right) \sqrt{\ln [1 /(2-2 \beta)]} & 0.5<\beta \leq 1\end{cases}
$$

$$
\sum_{i=1}^{n} x_{i} \mathrm{FN}\left(a_{i}^{1,2}, \sigma_{i}^{1,2}\right)=\mathrm{FN}\left(\sum_{i=1}^{n} x_{i}\left(\mathrm{P}_{i}^{1} * a_{i}^{1}+\mathrm{P}_{i}^{2} * a_{i}^{2}\right), \sum_{i=1}^{n} x_{i}\left(\mathrm{P}_{i}^{1} * \sigma_{i}^{1}+\mathrm{P}_{i}^{2} * \sigma_{i}^{2}\right)\right)
$$

$\mathrm{E}\left[\sum_{i=1}^{n} x_{i} \mathrm{FN}\left(a_{i}^{1,2}, \sigma_{i}^{1,2}\right)\right]=\sum_{i=1}^{n} x_{i}\left(\mathrm{P}_{i}^{1} * a_{i}^{1}+\mathrm{P}_{i}^{2} * a_{i}^{2}\right)$

$$
\operatorname{VaR}_{1-\beta}\left[\sum_{i=1}^{n} x_{i} \mathrm{FN}\left(a_{i}^{1,2}, \sigma_{i}^{1,2}\right)\right]= \begin{cases}\sum_{i=1}^{n} x_{i}\left[\left(\mathrm{P}_{i}^{1} * a_{i}^{1}+\mathrm{P}_{i}^{2} * a_{i}^{2}\right)+\left(\mathrm{P}_{i}^{1} * \sigma_{i}^{1}+\mathrm{P}_{i}^{2} * \sigma_{i}^{2}\right) \sqrt{\ln (1 / 2 \beta)}\right] & 0<\beta \leq 0.5 \\ \sum_{i=1}^{n} x_{i}\left(\mathrm{P}_{i}^{1} * a_{i}^{1}+\mathrm{P}_{i}^{2} * a_{i}^{2}\right)+\left(\mathrm{P}_{i}^{1} * \sigma_{i}^{1}+\mathrm{P}_{i}^{2} * \sigma_{i}^{2}\right) \sqrt{\ln [1 /(2-2 \beta)]} & 0.5<\beta \leq 1 .\end{cases}
$$




$$
\left\{\begin{array}{l}
\min \sum_{i=1}^{n} x_{i}\left[(2 \beta-1)\left(\mathrm{P}_{i}^{1} * a_{i}^{1}+\mathrm{P}_{i}^{2} * a_{i}^{2}\right)-2 \beta\left(\mathrm{P}_{i}^{1} * b_{i}^{1}+\mathrm{P}_{i}^{2} * b_{i}^{2}\right)\right] \\
\text { Subject to } \\
\quad \sum_{i=1}^{n} x_{i} \frac{\left(\mathrm{P}_{i}^{1} * a_{i}^{1}+\mathrm{P}_{i}^{2} * a_{i}^{2}\right)+\left(\mathrm{P}_{i}^{1} * b_{i}^{1}+\mathrm{P}_{i}^{2} * b_{i}^{2}\right)+\left(\mathrm{P}_{i}^{1} * c_{i}^{1}+\mathrm{P}_{i}^{2} * c_{i}^{2}\right)+\left(\mathrm{P}_{i}^{1} * d_{i}^{1}+\mathrm{P}_{i}^{2} * d_{i}^{2}\right)}{4} \geq R \\
\quad x_{1}+x_{2}+\cdots+x_{n}=1 \\
\quad x_{i} \geq 0, i=1,2, \cdots, n .
\end{array}\right.
$$

$\beta>0.5$,

$$
\begin{aligned}
& \int \min \sum_{i=1}^{n} x_{i}\left[(2 \beta-2)\left(\mathrm{P}_{i}^{1} * c_{i}^{1}+\mathrm{P}_{i}^{2} * c_{i}^{2}\right)-(2 \beta-1)\left(\mathrm{P}_{i}^{1} * d_{i}^{1}+\mathrm{P}_{i}^{2} * d_{i}^{2}\right)\right] \\
& \text { Subject to } \\
& \begin{array}{l}
\sum_{i=1}^{n} x_{i} \frac{\left(\mathrm{P}_{i}^{1} * a_{i}^{1}+\mathrm{P}_{i}^{2} * a_{i}^{2}\right)+\left(\mathrm{P}_{i}^{1} * b_{i}^{1}+\mathrm{P}_{i}^{2} * b_{i}^{2}\right)+\left(\mathrm{P}_{i}^{1} * c_{i}^{1}+\mathrm{P}_{i}^{2} * c_{i}^{2}\right)+\left(\mathrm{P}_{i}^{1} * d_{i}^{1}+\mathrm{P}_{i}^{2} * d_{i}^{2}\right)}{4} \geq R \\
x_{1}+x_{2}+\cdots+x_{n}=1 \\
x_{i} \geq 0, i=1,2, \cdots, n .
\end{array} \\
& \sum_{i=1}^{n} x_{i} \xi_{i}^{1,2}=\left(\sum_{i=1}^{n} x_{i}\left(\mathrm{P}_{i}^{1} * a_{i}^{1}+\mathrm{P}_{i}^{2} * a_{i}^{2}\right), \sum_{i=1}^{n} x_{i}\left(\mathrm{P}_{i}^{1} * b_{i}^{1}+\mathrm{P}_{i}^{2} * b_{i}^{2}\right), \sum_{i=1}^{n} x_{i}\left(\mathrm{P}_{i}^{1} * c_{i}^{1}+\mathrm{P}_{i}^{2} * c_{i}^{2}\right), \sum_{i=1}^{n} x_{i}\left(\mathrm{P}_{i}^{1} * d_{i}^{1}+\mathrm{P}_{i}^{2} * d_{i}^{2}\right)\right) \\
& \mathrm{E}\left[\xi_{i}^{1,2}\right]=\frac{\left(\mathrm{P}_{i}^{1} * a_{i}^{1}+\mathrm{P}_{i}^{2} * a_{i}^{2}\right)+\left(\mathrm{P}_{i}^{1} * b_{i}^{1}+\mathrm{P}_{i}^{2} * b_{i}^{2}\right)+\left(\mathrm{P}_{i}^{1} * c_{i}^{1}+\mathrm{P}_{i}^{2} * c_{i}^{2}\right)+\left(\mathrm{P}_{i}^{1} * d_{i}^{1}+\mathrm{P}_{i}^{2} * d_{i}^{2}\right)}{4} \\
& \mathrm{E}\left[\sum_{i=1}^{n} x_{i} \xi_{i}^{1,2}\right]=\sum_{i=1}^{n} x_{i} \frac{\left(\mathrm{P}_{i}^{1} * a_{i}^{1}+\mathrm{P}_{i}^{2} * a_{i}^{2}\right)+\left(\mathrm{P}_{i}^{1} * b_{i}^{1}+\mathrm{P}_{i}^{2} * b_{i}^{2}\right)+\left(\mathrm{P}_{i}^{1} * c_{i}^{1}+\mathrm{P}_{i}^{2} * c_{i}^{2}\right)+\left(\mathrm{P}_{i}^{1} * d_{i}^{1}+\mathrm{P}_{i}^{2} * d_{i}^{2}\right)}{4} . \\
& \mathrm{VaR}_{1-\beta}= \begin{cases}\sum_{i=1}^{n} x_{i}\left[(2 \beta-1)\left(\mathrm{P}_{i}^{1} * a_{i}^{1}+\mathrm{P}_{i}^{2} * a_{i}^{2}\right)-2 \beta\left(\mathrm{P}_{i}^{1} * b_{i}^{1}+\mathrm{P}_{i}^{2} * b_{i}^{2}\right)\right] & 0<\beta \leq 0.5 \\
\sum_{i=1}^{n} x_{i}\left[(2 \beta-2)\left(\mathrm{P}_{i}^{1} * c_{i}^{1}+\mathrm{P}_{i}^{2} * c_{i}^{2}\right)-(2 \beta-1)\left(\mathrm{P}_{i}^{1} * d_{i}^{1}+\mathrm{P}_{i}^{2} * d_{i}^{2}\right)\right] & 0.5<\beta \leq 1 .\end{cases}
\end{aligned}
$$

$$
\left\{\begin{array}{l}
\min \sum_{i=1}^{n} x_{i}\left[(2 \beta-1)\left(\mathrm{P}_{i}^{1} * a_{i}^{1}+\mathrm{P}_{i}^{2} * a_{i}^{2}\right)-2 \beta\left(\mathrm{P}_{i}^{1} * a_{i}^{1}+\mathrm{P}_{i}^{2} * a_{i}^{2}\right)\right] \\
\text { Subject to } \\
\quad \sum_{i=1}^{n} x_{i} \frac{\left(\mathrm{P}_{i}^{1} * a_{i}^{1}+\mathrm{P}_{i}^{2} * a_{i}^{2}\right)+2\left(\mathrm{P}_{i}^{1} * b_{i}^{1}+\mathrm{P}_{i}^{2} * b_{i}^{2}\right)+\left(\mathrm{P}_{i}^{1} * c_{i}^{1}+\mathrm{P}_{i}^{2} * c_{i}^{2}\right)}{4} \geq R \\
\quad x_{1}+x_{2}+\cdots+x_{n}=1 \\
\quad x_{i} \geq 0, i=1,2, \cdots, n .
\end{array}\right.
$$

$\beta>0.5$,

$$
\left\{\begin{array}{l}
\min \sum_{i=1}^{n} x_{i}\left[(2 \beta-2)\left(\mathrm{P}_{i}^{1} * b_{i}^{1}+\mathrm{P}_{i}^{2} * b_{i}^{2}\right)-(2 \beta-1)\left(\mathrm{P}_{i}^{1} * c_{i}^{1}+\mathrm{P}_{i}^{2} * c_{i}^{2}\right)\right] \\
\text { Subject to } \\
\quad \sum_{i=1}^{n} x_{i} \frac{\left(\mathrm{P}_{i}^{1} * a_{i}^{1}+\mathrm{P}_{i}^{2} * a_{i}^{2}\right)+2\left(\mathrm{P}_{i}^{1} * b_{i}^{1}+\mathrm{P}_{i}^{2} * b_{i}^{2}\right)+\left(\mathrm{P}_{i}^{1} * c_{i}^{1}+\mathrm{P}_{i}^{2} * c_{i}^{2}\right)}{4} \geq R \\
\quad x_{1}+x_{2}+\cdots+x_{n}=1 \\
\quad x_{i} \geq 0, i=1,2, \cdots, n .
\end{array}\right.
$$


to find the optimal solution in a possible space. If the position of a given particle can produce a better result, the other particles will approach the given particle.

The PSO algorithm can find the optimal solution in fewer iterations than other algorithms, but the PSO algorithm suffers from the local convergence problem. Therefore, in our previous study [17], we developed the concepts of escape speed (ES) and particle restart position (PRP) and used them to improve the original PSO algorithm. In this study, we maintain these improvements to mitigate the local convergence problem. You may refer to [17] for detailed information.

Suppose that there is a $k$-security portfolio selection problem and that the swarm of PSO has $n$ particles with $i$ and $j$ denoting the ith particle and the $j$ th security. Then, the FBS-PSO algorithm of this research can be summarized as follows:

[Step 1]. Initialize the investment proportions: The initial values of the investment proportions of each security are set at random in this system. We generate a group of random values $\left(a_{1}, \cdots, a_{e}, \cdots, a_{k}\right)$ that comprise a total value called the 'sum', and divide each of the random values by the sum. As a result, we obtain a set of values $\left(x_{1}, \cdots, x_{e}, \cdots, x_{k}\right)$ that are computed from $x_{e}=a_{e} /$ sum. Clearly, $x_{e}$ is always within $(0,1)$ and the sum is identically equal to 1 .

[Step 2]. Check the profit and risk levels: The $x_{e}$ values obtained in Step 1 may not satisfy the profit or risk constraints of models (15) and (16). For example in model (16), the expected value of security returns $\mathrm{E}\left[x_{1} \xi_{1}^{1,2}+\cdots+x_{e} \xi_{e}^{1,2}+\right.$ $\left.\cdots+x_{n} \xi_{n}^{1,2}\right]$ must not be less than $\mathrm{R}$; if the result is smaller than our goal, we repeat the above processes until the coefficients satisfy all of the conditions. These accepted coefficients are then taken as the initial particles position. The position for particle $i$ is recorded in the array particle $[i][j]$.

[Step 3]. Initialize the personal best (pbest $[i][j])$ and the global best (gbest $[t][j]$ ), where $t$ denotes the current iteration of the swarm. After initializing each particle, set pbest $[i][j]=$ particle $[i][j]$. Then, calculate the VaR of each particle using the fuzzy birandom simulation and save these values in the array VaR[0][i]. Suppose particle ' $b$ ' minimizes the VaR; then, set gbest $[0][j]=$ particle $[b][j]$. After generating the particles' velocities $v[i][j]$, the initialization process is complete.

[Step 4]. Particle iterations: The particle positions are updated using the following equation,

$$
\begin{aligned}
& v[i][j]=w * v[i][j] \\
& +c 1 * \operatorname{random}(0,1) *(\text { pbest }[i][j]-\operatorname{particle}[i][j]) \\
& +c 2 * \operatorname{random}(0,1) *(\operatorname{gbest}[t][j]-\text { particle }[i][j]),
\end{aligned}
$$

$$
\text { particle }[i][j]=\text { particle }[i][j]+v[i][j] .
$$

In Eq. (37), $c 1$ and $c 2$ are learning rates, which are normally set to 2 , and $w$ is the speed weight.

[Step 5]. Check the feasibility of the new positions. The initialization task in Step 1 should be maintained here to check the feasibility: Take the sum of particle $[i][j]$ as sum $=$ $\sum_{j=1}^{k}$ particle $[i][j]$, particle $[i][j]=$ particle $[i][j] /$ sum ; then check whether the expected value is acceptable. If so, compute the VaR values and compare them (the smaller the better). Apply a transverse comparison to update pbest $[i][j]$ and use the total comparison to modify gbest $[t][j]$. Again, $t$ denotes the iteration.

[Step 6]. Iterations: Run the particles for $T$ iterations, where the final optimal solution is gbest $[T][j]$, which indicates how much should be invested for each security. Over the course of these iterations, we use the improvements in a previous study [17] to mitigate the local convergence problem.

\section{Numerical Examples}

We provide the following numerical example to illustrate the proposed model and the solution algorithm. All of the experiments were implemented with C code on a Dell E8500 $3.16 \mathrm{GHz}-\mathrm{CPU}$ personal computer. We also discuss the results of the FBVaR-PSM with some previous methods.

\subsection{The Performance of the FBVaR-PSM and FBS-PSO}

Suppose that there are 20 securities whose possible returns are measured by fuzzy birandom variables. We employ different distributed fuzzy variables to describe these securities, since their returns are affected by different factors and could be in different distributions. In this paper, we assume that the first 10 security returns are triangular distribution as $(\mathrm{a}, \mathrm{b}, \mathrm{c})$ while the other 10 are Gaussian distribution as $\mathrm{FN}\left(\mu, \sigma^{2}\right)$. The rise and fall probabilities of each security are obtained based on historical data listed in Table 2 .

Example 6.1 For risk-lovers [model (16)], supposing the expected value of the investment should not be less than 0.35 and $\beta=0.1$. Consequently, the following special case is formed:

$$
\left\{\begin{array}{l}
\min \operatorname{VaR}_{0.9}=\sup \{\lambda \mid \operatorname{Ch}(\mathcal{L} \geq \lambda) \geq 0.1\} \\
\text { Subject to } \\
\quad \mathrm{E}\left[x_{1} \xi_{1}^{1,2}+x_{2} \xi_{2}^{1,2}+\cdots+x_{20} \xi_{20}^{1,2}\right] \geq 0.35 \\
x_{1}+x_{2}+\cdots+x_{20}=1 \\
x_{i} \geq 0, i=1,2, \cdots, 20
\end{array}\right.
$$

Respectively, $\mathcal{L}=-\left(x_{1} \xi_{1}^{1,2}+x_{2} \xi_{2}^{1,2}+\cdots+x_{20} \xi_{20}^{1,2}\right)$ and the confidence level is 0.9 .

Then, we use the FBS-PSO algorithm to find the optimal solution. The parameters are set as follows: particle No. $=10, w=0.5, c 1=2, c 2=2$. After 300 iterations, the greatest loss under confidence level 0.9 is 0.068 while the expected return is 0.369 , and the invested money is allocated as in Fig. 2.

Example 6.2 For risk-averters [model (15)], supposing the VaR is not larger than 0.35 and confidence level is still taken as 0.9. We can obtain the following specific case: 
Table 2 Security return.

\begin{tabular}{cccccc}
\hline Security No. & Probability & Return & Security No. & Probability & Return \\
1 & $\mathrm{P}_{1}^{1}: 0.56$ & $(-0.7,-0.3,0)$ & 11 & $\mathrm{P}_{11}^{1}: 0.57$ & $\mathrm{FN}(-0.3,0.5)$ \\
& $\mathrm{P}_{1}^{2}: 0.44$ & $(0,0.6,1.8)$ & & $\mathrm{P}_{1:}^{2}: 0.43$ & $\mathrm{FN}(0.8,0.4)$ \\
\hline 2 & $\mathrm{P}_{2}^{1}: 0.38$ & $(-0.8,-0.4,0)$ & 12 & $\mathrm{P}_{12}^{1}: 0.46$ & $\mathrm{FN}(-0.3,0.6)$ \\
& $\mathrm{P}_{2}^{2}: 0.62$ & $(0,0.4,2.0)$ & & $\mathrm{P}_{12}^{2}: 0.54$ & $\mathrm{FN}(0.9,0.5)$ \\
\hline 3 & $\mathrm{P}_{3}^{1}: 0.50$ & $(-1.1,-0.6,0)$ & 13 & $\mathrm{P}_{13}^{1}: 0.39$ & $\mathrm{FN}(-0.4,0.3)$ \\
& $\mathrm{P}_{3}^{2}: 0.50$ & $(0,0.8,2.8)$ & & $\mathrm{P}_{13}^{2}: 0.61$ & $\mathrm{FN}(0.9,0.4)$ \\
\hline 4 & $\mathrm{P}_{4}^{1}: 0.33$ & $(-1.6,-0.8,0)$ & 14 & $\mathrm{P}_{14}^{1}: 0.45$ & $\mathrm{FN}(-0.3,0.4)$ \\
& $\mathrm{P}_{4}^{2}: 0.67$ & $(0,0.9,3.2)$ & & $\mathrm{P}_{14}^{2}: 0.55$ & $\mathrm{FN}(0.8,0.5)$ \\
\hline 5 & $\mathrm{P}_{5}^{1}: 0.28$ & $(-1.4,-0.8,0)$ & 15 & $\mathrm{P}_{15}^{1}: 0.48$ & $\mathrm{FN}(-0.2,0.7)$ \\
& $\mathrm{P}_{5}^{2}: 0.72$ & $(0,0.8,3.9)$ & & $\mathrm{P}_{15}^{2}: 0.52$ & $\mathrm{FN}(1.0,0.8)$ \\
\hline 6 & $\mathrm{P}_{6}^{1}: 0.55$ & $(-0.9,-0.6,0)$ & 16 & $\mathrm{P}_{16}^{1}: 0.49$ & $\mathrm{FN}(-0.5,0.3)$ \\
& $\mathrm{P}_{6}^{2}: 0.45$ & $(0,0.8,2.2)$ & & $\mathrm{P}_{16}^{2}: 0.51$ & $\mathrm{FN}(0.3,0.6)$ \\
\hline 7 & $\mathrm{P}_{7}^{1}: 0.45$ & $(-1.2,-0.7,0)$ & 17 & $\mathrm{P}_{17}^{1}: 0.38$ & $\mathrm{FN}(-0.3,0.7)$ \\
& $\mathrm{P}_{7}^{2}: 0.55$ & $(0,0.9,1.9)$ & & $\mathrm{P}_{17}^{2}: 0.62$ & $\mathrm{FN}(0.8,0.7)$ \\
\hline 8 & $\mathrm{P}_{8}^{1}: 0.49$ & $(-1.4,-0.6,0)$ & 18 & $\mathrm{P}_{18}^{1}: 0.50$ & $\mathrm{FN}(-0.4,0.5)$ \\
& $\mathrm{P}_{8}^{2}: 0.51$ & $(0,1.1,3.2)$ & & $\mathrm{P}_{18}^{2}: 0.50$ & $\mathrm{FN}(0.7,0.4)$ \\
\hline 9 & $\mathrm{P}_{0}^{1}: 0.29$ & $(-1.5,-0.4,0)$ & 19 & $\mathrm{P}_{19}^{1}: 0.43$ & $\mathrm{FN}(-0.3,0.6)$ \\
& $\mathrm{P}_{9}^{2}: 0.71$ & $(0,0.9,2.8)$ & & $\mathrm{P}_{19}^{2}: 0.57$ & $\mathrm{FN}(0.8,0.6)$ \\
\hline 10 & $\mathrm{P}_{10}^{1}: 0.44$ & $(-1.0,-0.3,0)$ & 20 & $\mathrm{P}_{20}^{1}: 0.46$ & $\mathrm{FN}(-0.4,0.6)$ \\
& $\mathrm{P}_{10}^{2}: 0.56$ & $(0,0.8,2.5)$ & & $\mathrm{P}_{20}^{2}: 0.54$ & $\mathrm{FN}(0.8,0.7)$ \\
\hline
\end{tabular}

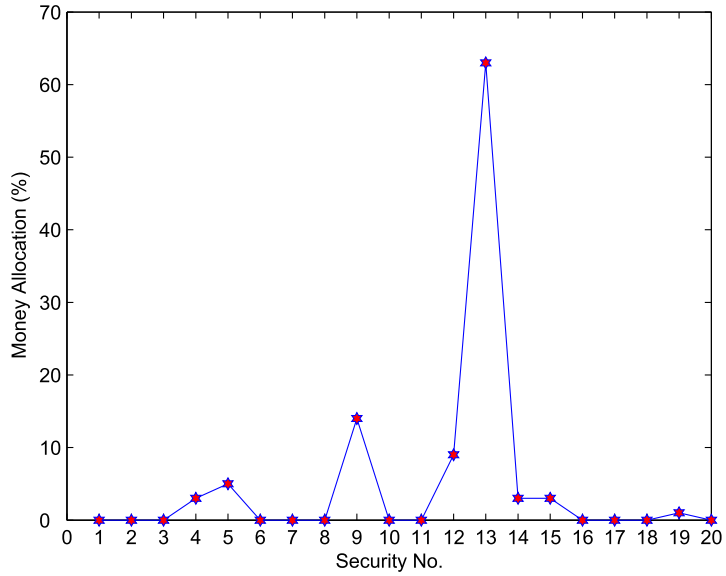

Fig. 2 Optimal solution of the risk-lovers model.

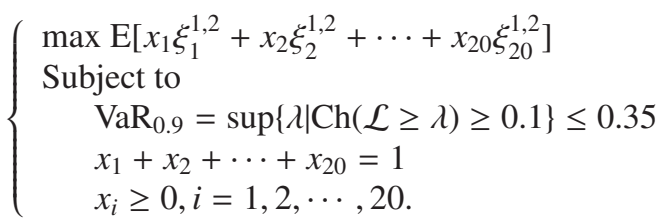

Then, after 300 iterations, the largest expect return under confidence level 0.9 is 0.732 while the largest investment loss is 0.272 , and invest money should be allocated as Fig. 3 shows.

\subsection{Discussion on the Optimal Results of the FBVaR-PSM}

\subsubsection{Experimental Results Analysis}

Based on Example 6.1, we first analyze the relationship between the investment profit and the investment risk. Figure 4 shows the sensitivity of the fuzzy birandom VaR to different expected values.

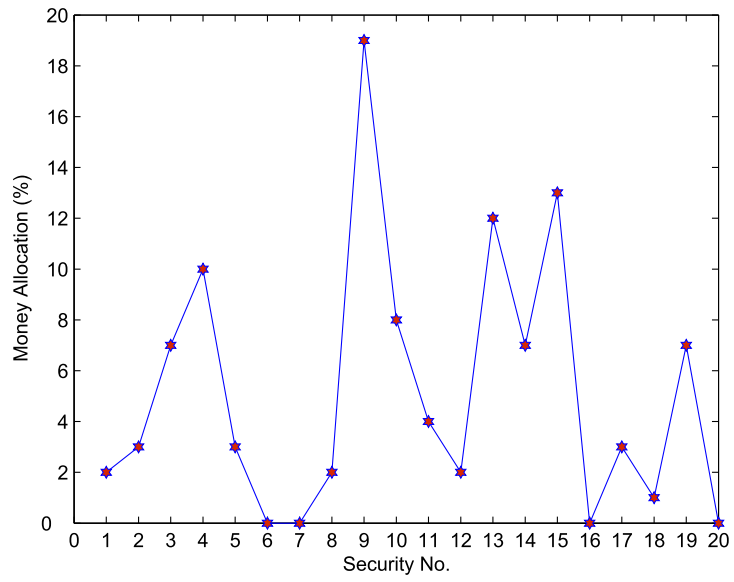

Fig. 3 Optimal solution of risk-averters model.

When we increase the expected value, the VaR becomes larger. This is reasonable: higher returns are always accompanied by higher risk. If we use a high expected level, then the final optimal solution for a portfolio must focus on some securities with higher returns, which always include higher risk. For example, in Fig. 4, the potential risk of a profit level of 0.45 is much higher than the risk for lower profit levels. Therefore, the investor should set an appropriate expected value that can be satisfied without causing too much potential loss.

\subsubsection{Discussions of Previous Portfolio Selection Methods}

Compared with previous research, this study differs in two ways: First, the selection results here are obtained using a technical analysis based on fuzzy birandom variables, while the fuzzy variables are generally used in conventional methods. Second, the fuzzy birandom VaR is applied to measure the exact potential loss as the investment risk, while most of 


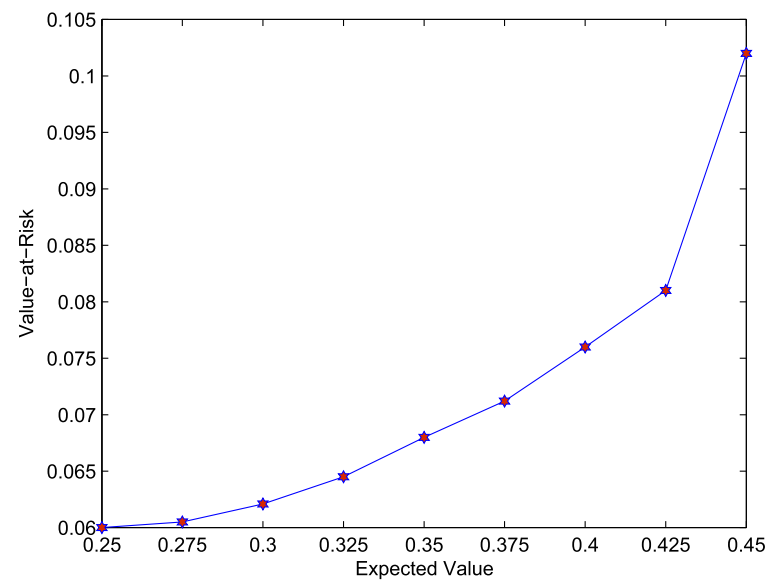

Fig. 4 The sensitivity between VaR and expected value.

the previous investment risk measurements aim to enhance portfolio stability.

From a forecasting perspective, we can see that the security returns of Table 2 are provided based on the historical rising and falling probabilities, and the possibility distributions of the past transaction prices. This feature helps investors distinguish the advanced securities (the probability of a rising price is higher than that of a falling price) among a series of candidates and makes the forecasting more approximate to real values than using only the fuzzy variables.

From the selection results perspective, the fuzzy birandom $\mathrm{VaR}$ is a more sensitive analysis for general investors than other measurements. It is known that investors are well-informed of the purposes of the existing risk measurements, such as mean-variance, mean-semivariance or entropy; minimizing these values can improve selection stability. However, general investors cannot identify the potential risk included in these numerical values. As we have explained in Sect. III A, these approaches are not sensitive to measuring future risk, as a result,investors cannot clearly determine the parameters of these approaches and thus cannot evaluate the results of these models. However,in our study, investors can determine the confidence levels and the expected returns as they see fit, and any change in these values will be reflected by the numerical values of the fuzzy birandom VaR. Therefore, the proposed model FBVaR-PSM can provide investors with more effective information and help them make decisions easier than non-VaR approaches.

\section{Conclusion}

In this paper, a technical analysis based on fuzzy birandom variables was employed to build a new portfolio selection model, called FBVaR-PSM. The quantitative risk measurement VaR was extended to a fuzzy birandom case to measure the exact loss of a portfolio. Compared to most of the existing fuzzy portfolio selection models, the FBVaR-PSM is superior to conventional approaches for both forecasting and risk measurement. To solve the model, we provided several theorems and a fuzzy birandom simulation-based PSO algorithm, called the FBS-PSO. The improvement of the PSO algorithm proposed in our previous study was maintained here to mitigate the local convergence problem. The FBVaR-PSM and FBS-PSO were applied to solve two numerical examples based on different risk attitudes. Finally, we analyzed the selection results and discussed this research with reference to previous studies. In future work, we will extend the FBVaR-PSM to the multi-objective problem, and we will apply the model and solution method to some realworld applications. In addition, the fuzzy birandom method discussed in this paper is applicable to other investment problems.

\section{Acknowledgements}

This research is partly supported by "Ambient SoC Global Program of Waseda University" of the Ministry of Education, Culture, Sports, Science and Technology, Japan.

Bo Wang is supported by the Research Fellowships of the Japan Society for the Promotion of Science (JSPS) for Young Scientists.

This work is supported in part by Grants-in-Aid for Scientific Research, MEXT (No.23500289).

\section{References}

[1] H. Markowitz, "Portfolio selection," J. Finance, vol.7, no.1, pp.7791, 1952.

[2] X. Huang, "Mean-entropy models for fuzzy portfolio selection," IEEE Trans. Fuzzy Syst., vol.16, no.4, pp.1096-1101, 2008.

[3] X. Huang, "Mean-semivariance models for fuzzy portfolio selection," J. Computational and Applied Mathematics, vol.217, no.1, pp.1-8, 2008.

[4] D. Dubois and H. Prade, Possibility Theory, Plenum Press, New York, 1988.

[5] D. Duffie and J. Pan, “An overview of value-at-risk," J. Derivatives, vol.4, no.3, pp.7-49, 1997.

[6] P. Jorion, Value at Risk: The New Benchmark for Controlling Market Risk, McGraw-Hill, 2000.

[7] J. Kennedy and R.C. Eberhaart, "Particle swarm optimization," Proc. 1995 IEEE International Conference on Neual Network, IV, pp.1942-1948, 1995.

[8] B. Liu and Y.K. Liu, "Expected value of fuzzy variable and fuzzy expected value models," IEEE Trans. Fuzzy Syst., vol.10, no.4, pp.445-450, 2002.

[9] J. Watada, "Fuzzy portfolio selection and its application to decision making," Tatra Mountains Math. Publication, vol.13, no.4, pp.219248, 1997.

[10] R.J. Almeida and U. Kaymak, "Probabilistic fuzzy systems in valueat-risk estimation," Int. J. Intelligent Systems in Accounting, Finance and Management, vol.16, no.1/2, pp.49-70, 2009.

[11] X. Huang, "Risk curve and fuzzy portfolio selection," Computers and Mathematics with applications, vol.55, no.6, pp.1102-1112, 2008.

[12] S. Wang and J. Watada, "Two-stage fuzzy stochastic programming with Value-at-risk criteria," Applied Soft Computing, vol.11, no.1, pp.1044-1056, 2011.

[13] S. Wang, J. Watada, and W. Pedrycz, "Value-at-risk-based two-satge fuzzy facility location problems," IEEE Trans. Industrial Informatics, vol.5, no.4, pp.465-482, 2009.

[14] X. Li, Z. Qin, and S. Kar, "Mean-variance-skewness model for portfolio selection with fuzzy returns," European Journal of Operational Research, vo1.202, no.1, pp.239-247, 2009. 
[15] Z. Zmeskal, "Value at risk methodology of international index portfolio under soft conditions," International Review of Financial Analysis, vol.14, no.6, pp.263-275, 2005.

[16] R.A. Jabr, "Robust self-scheduling under price uncertainty using conditional value-at-risk," IEEE Trans. Power Syst., vol.20, no.4, pp.1852-1858, 2005.

[17] B. Wang, S. Wang, and J. Watada, "Fuzzy portfolio selection models with value-at-risk," IEEE Trans. Fuzzy Syst., vol.19, no.4, pp.758$769,2011$.

[18] A. Charnes and W.W. Cooper, "Chance-constrained programming," Management Science, vol.6, no.1, pp.73-79, 1959.

[19] J. Peng and B. Liu, "Birandom variables and birandom programming," Computers \& Industrial Engineering, vol.53, no.3, pp.433453, 2007.

[20] Y.K. Liu and B. Liu, "Fuzzy random variable: A scalar expected value operator," Fuzzy Optimization and Decision Making, vol.2, no.2, pp.143-160, 2003.

[21] M. Douglas, Trading in The Zone, Prentice Hall, New York, 2000.

[22] C.D. Kirkpatrick and J.R. Dahlquist, Technical Analysis: The Complete Resource for Financial Market Technicians, Financial Times Press, 2006.

[23] Y.K. Liu and B. Liu, "On minimum-risk problems in fuzzy random decision systems," Computers \& Operations Research, vol.32, no.2, pp.257-283, 2005.

[24] Y.K. Liu, "Convergent results about the use of fuzzy simulation in fuzzy optimization problems," IEEE Trans. Fuzzy Syst., vol.14, no.2, pp.295-304, 2006.

[25] M.H.A. Davis and A.R. Norman, "Portfolio selection with transaction costs," Mathematics of Operations Research, vol.15, no.4, pp.676-713, 1990.

[26] O. Ledoit and M. Wolf, "Improved estimation of the covariance matrix of stock returns with an application to portfolio selection," J. Empirical Finance, vol.10, no.5, pp.603-621, 2003.

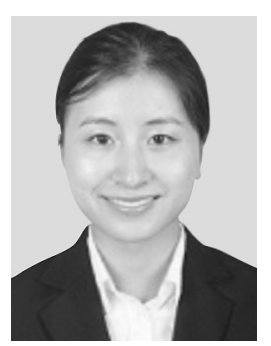

You $\mathbf{L i}$ received the B.Sc. degree from Nanjing Normal University, Nanjing, China and the M.Sc. degree from Waseda University, Japan. She is currently working towards the Ph.D. degree at the Graduate School of Information, Production and Systems of Waseda University, Japan. Her research interests include management engineering and multi-objective optimization problems.

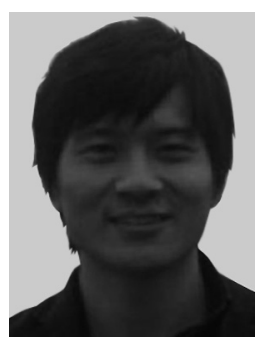

Bo Wang received the B.Sc. degree in software engineering from Southeast University, Nanjing, China and the M.Sc. and Ph.D. degrees from the Graduate School of Information, Production and Systems, Waseda University, Japan. He was a Research Assistant of the Global COE Program, Waseda University, Ministry of Education, Culture, Sports, Science and Technology, Japan (2009.10-2012.3). He is currently a Special Research Fellow of the Japan Society for the Promotion of Science (JSPS). His research interests include soft computing, management engineering, power supply systems and Meta-heuristic algorithms.

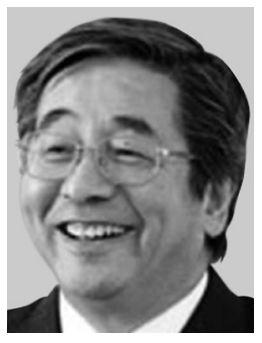

Junzo Watada received the B.Sc. and M.Sc. degrees in electrical engineering from Osaka City University, Osaka, Japan, and the Ph.D. degree from Osaka Prefecture University, Osaka. Currently, he is a Professor of Management Engineering, Knowledge Engineering and Soft Computing at the Graduate School of Information, Production Systems, Waseda University, Fukuoka, Japan. His professional interests include soft computing, tracking system, knowledge engineering, and management engineering. Prof. Watada is a recipient of the Henri Coanda Medal Award from Invention in Romania in 2002. He is a Life Fellow of Japan Society for Fuzzy Theory and intelligent Informatics (SOFT). He is the Principal Editor, a Co-Editor, and an Associate Editor of various international journals, including the International Journal of Intelligent Decision Technology, ICIC Express Letters, and the IMECHE Journal of Systems and Control Engineering, Fuzzy Optimization Decision Making, Information Science and IEEE SMC 